\title{
Hunting inflatons at FASER
}

\author{
Nobuchika Okada ${ }^{1, *}$ and Digesh Raut $\odot^{2, \dagger}$ \\ ${ }^{1}$ Department of Physics and Astronomy, University of Alabama, Tuscaloosa, Alabama 35487, USA \\ ${ }^{2}$ Bartol Research Institute, Department of Physics and Astronomy, University of Delaware, \\ Newark, Delaware 19716, USA
}

(Received 28 October 2019; accepted 5 March 2021; published 29 March 2021)

\begin{abstract}
We consider a nonminimal quartic inflation scenario in the minimal $\mathrm{U}(1)_{X}$ extension of the Standard Model (SM) with the classical conformal invariance, where the inflaton is identified with the $\mathrm{U}(1)_{X}$ Higgs field $(\phi)$. By virtue of the classically conformal invariance and the radiative $\mathrm{U}(1)_{X}$ symmetry breaking via the Coleman-Weinberg mechanism, the inflationary predictions (in particular, the tensor-to-scaler ratio $r$ ), the $\mathrm{U}(1)_{X}$ coupling $g_{X}$, and the $\mathrm{U}(1)_{X}$ gauge boson mass $m_{Z^{\prime}}$ are all determined by only two free parameters: the inflaton mass $m_{\phi}$ and its mixing angle $\theta$ with the SM Higgs field. FASER can search for a long-lived scalar, which is the inflaton in our scenario, for the parameter ranges $0.1 \lesssim m_{\phi}[\mathrm{GeV}] \lesssim 4$ and $10^{-5} \lesssim \theta \lesssim 10^{-3}$. Therefore, if such a scalar is discovered at FASER, both $m_{\phi}$ and $\theta$ would be fixed, leading to the predictions for $r, g_{X}$, and $m_{Z^{\prime}}$ in our model. These predictions can be tested by future cosmological observations and LHC searches for the $Z^{\prime}$ boson resonance.
\end{abstract}

DOI: 10.1103/PhysRevD.103.055022

\section{INTRODUCTION}

Very recently, the ForwArd Search ExpeRiment (FASER) [1] was approved to search for light, weakly interacting, electrically neutral long-lived particles at the Large Hadron Collider (LHC). Such long-lived particles are included in a variety of new physics models beyond the Standard Model (SM). In the experiment, a detector will be located along the beam trajectory 480 meters downstream from the interaction point within the ATLAS detector at the LHC. This setup is designed to search for light, long-lived particles with the following advantages. (i) The HighLuminosity upgrade of the LHC (HL-LHC) can produce a huge number of hadrons in the forward region, which could decay into light long-lived particles. Even if such a decay process is extremely rare, the huge number of produced hadrons provides us with a sizable number of events for the long-lived particle production. (ii) Such light particles are highly boosted in the beam direction and mostly produced in the forward region. (iii) Because of very weak interactions, such particles can have a decay length of $\mathcal{O}(100 \mathrm{~m})$. The displaced vertex signature from such long-lived particles is almost free from the SM backgrounds. In Refs. [2,3], the

\footnotetext{
*okadan@ua.edu

'draut@udel.edu
}

Published by the American Physical Society under the terms of the Creative Commons Attribution 4.0 International license. Further distribution of this work must maintain attribution to the author(s) and the published article's title, journal citation, and DOI. Funded by SCOAP. authors explored the possibility of detecting an SM singlet scalar $(\phi)$ at FASER and other proposed experiments for the displaced vertex search. The singlet scalar only couples with the SM particles through its mixing with the SM Higgs boson. Hence, the production rate and lifetime of the particle $\phi$ are controlled by only two parameters: its mass $m_{\phi}$ and mixing angle $\theta$ with the SM Higgs field. Impressively, these experiments are capable of probing extremely small mixing angles, $10^{-7} \lesssim \theta \lesssim 10^{-3}$, for $0.1 \lesssim m_{\phi}[\mathrm{GeV}] \lesssim 10[2,3]$.

The authors of the pioneering work [4] pointed out that the long-lived light scalar can be identified with a light inflaton in their inflation model. Once observed, its mass and mixing angle with the SM Higgs field can be measured. In their model, the measured mass and mixing angle correspond to the inflationary predictions. Although the quartic inflation scenario discussed in Ref. [4] is excluded by Planck 2018 results [5], it can be made consistent by including nonminimal gravitational coupling [6].

In this paper we consider nonminimal quartic inflation in a classically conformal $\mathrm{U}(1)_{X}$ extended $\mathrm{SM}$, which the authors of the present paper have proposed with their collaborators [7] (see also Ref. [8]). By imposing conformal invariance at the classical level on the minimal U(1) ${ }_{X}$ extended SM [9], all of the mass terms in the Higgs potential are forbidden. As a result, the $\mathrm{U}(1)_{X}$ gauge symmetry is radiatively broken by the Coleman-Weinberg (CW) mechanism [10], which subsequently drives the electroweak symmetry breaking through a mixing quartic coupling between the $\mathrm{U}(1)_{X}$ Higgs and SM Higgs fields [11]. As first pointed out in Ref. [12], the classical conformal invariance could be a clue to solving the gauge hierarchy problem of the SM. In our paper [7], 
we identified the $\mathrm{U}(1)_{X}$ Higgs field with a nonminimal gravitational coupling as the inflaton. Because of the classically conformal invariance, this scenario not only leads to inflationary predictions consistent with the Planck 2018 results [5], but also provides a direct connection between the inflationary predictions and the LHC search for the $\mathrm{U}(1)_{X}$ gauge boson $\left(Z^{\prime}\right)$ resonance. In our previous work [7], the scalar sector was not analyzed in detail from the viewpoint of collider phenomenology. The main purpose of this paper is to point out that if the inflaton mass and its mixing angle with the SM Higgs field lie in a suitable range, the inflaton can be searched by FASER with a direct connection to the inflationary predictions. ${ }^{1}$ Therefore, three independent experiments - namely, the inflaton search at FASER, the $Z^{\prime}$ boson resonance search at the HL-LHC, and the precision measurement of the inflationary predictions-are complementary tests of our inflation scenario.

\section{CLASSICALLY CONFORMAL U(1) $)_{X}$ MODEL}

We first define our model with the particle content listed in Table I, where the $\mathrm{U}(1)_{X}$ charge of a particle is defined as a linear combination of its SM hypercharge and its $B-L$ (baryon minus lepton) number. The $\mathrm{U}(1)_{X}$ charges are determined by a real parameter, $x_{H}$, and the well-known minimal $\mathrm{U}(1)_{B-L}$ model [14] is realized in the limit $x_{H} \rightarrow 0$. In the presence of the three right-hand neutrinos (RHNs) $N_{R}^{1,2,3}$, this model is free from all of the gauge and mixed gauge-gravitational anomalies. Once the $\mathrm{U}(1)_{X}$ Higgs field $(\Phi)$ develops a vacuum expectation value (VEV), $\langle\Phi\rangle=v_{X} / \sqrt{2}$, the $\mathrm{U}(1)_{X}$ gauge symmetry is broken and the $Z^{\prime}$ boson becomes massive, $m_{Z^{\prime}}=2 g_{X} v_{X}$, where $g_{X}$ is the $\mathrm{U}(1)_{X}$ gauge coupling.

The Yukawa sector of the SM is extended to include

$$
\mathcal{L}_{Y} \supset-\sum_{i, j=1}^{3} Y_{D}^{i j} \overline{\ell_{L}^{i}} H N_{R}^{j}-\frac{1}{2} \sum_{k=1}^{3} Y_{M}^{k} \Phi \overline{N_{R}^{k C}} N_{R}^{k}
$$

where $Y_{D}\left(Y_{M}\right)$ is a Dirac (Majorana) type Yukawa coupling. Without loss of generality, we chose the Majorana Yukawa couplings to be flavor diagonal. The Majorana masses for the RHNs are generated by the $\mathrm{U}(1)_{X}$ gauge symmetry breaking. For simplicity, we fix $Y_{M}^{1,2,3}=$ $Y_{M}$ and thus RHNs have a degenerate mass spectrum, $m_{N}=Y_{M} v_{X} / \sqrt{2}$. After the electroweak symmetry breaking, the light neutrino masses are generated via the type-I seesaw mechanism [15].

Imposing the classical conformal invariance, the Higgs potential of our model is given by

$$
V=\lambda_{H}\left(H^{\dagger} H\right)^{2}+\lambda_{\Phi}\left(\Phi^{\dagger} \Phi\right)^{2}-\lambda_{\text {mix }}\left(H^{\dagger} H\right)\left(\Phi^{\dagger} \Phi\right),
$$

\footnotetext{
${ }^{1}$ See Ref. [13] for a similar work for an axion-like particle as the inflaton.
}

TABLE I. The particle content of the minimal $\mathrm{U}(1)_{X}$ model. $i=1,2,3$ is the generation index.

\begin{tabular}{ccccc}
\hline \hline & $\mathrm{SU}(3)_{c}$ & $\mathrm{SU}(2)_{L}$ & $\mathrm{U}(1)_{Y}$ & $\mathrm{U}(1)_{X}$ \\
\hline$q_{L}^{i}$ & $\mathbf{3}$ & $\mathbf{2}$ & $1 / 6$ & $(1 / 6) x_{H}+(1 / 3)$ \\
$u_{R}^{i}$ & $\mathbf{3}$ & $\mathbf{1}$ & $2 / 3$ & $(2 / 3) x_{H}+(1 / 3)$ \\
$d_{R}^{i}$ & $\mathbf{3}$ & $\mathbf{1}$ & $-1 / 3$ & $(-1 / 3) x_{H}+(1 / 3)$ \\
$\ell_{L}^{i}$ & $\mathbf{1}$ & $\mathbf{2}$ & $-1 / 2$ & $(-1 / 2) x_{H}-1$ \\
$e_{R}^{i}$ & $\mathbf{1}$ & $\mathbf{1}$ & -1 & $-x_{H}-1$ \\
$H$ & $\mathbf{1}$ & $\mathbf{2}$ & $-1 / 2$ & $(-1 / 2) x_{H}$ \\
$N_{R}^{i}$ & $\mathbf{1}$ & $\mathbf{1}$ & 0 & -1 \\
$\Phi$ & $\mathbf{1}$ & $\mathbf{1}$ & 0 & 2 \\
\hline \hline
\end{tabular}

where we set $\lambda_{H, \Phi \text {,mix }}>0$. Assuming $\lambda_{\text {mix }} \ll 1$ (this will be justified later), we can separately analyze the Higgs potential for $\Phi$ and $H .^{2}$ The CW potential for the Higgs field $\Phi$ at the one-loop level is given by [10]

$$
V(\phi)=\frac{\lambda_{\Phi}}{4} \phi^{4}+\frac{\beta_{\Phi}}{8} \phi^{4}\left(\ln \left[\frac{\phi^{2}}{v_{X}^{2}}\right]-\frac{25}{6}\right)
$$

where $\phi=\sqrt{2} \Re[\Phi], v_{\mathrm{X}}$ is chosen as a renormalization scale, and the coefficient of the one-loop corrections is approximately given by

$$
16 \pi^{2} \beta_{\Phi} \simeq 96 g_{X}^{4}-3 Y_{M}^{4}
$$

The stationary condition, $d V /\left.d \phi\right|_{\phi=v_{X}}=0$, leads to

$$
\overline{\lambda_{\Phi}}=\frac{11}{6} \overline{\beta_{\Phi}},
$$

where the barred quantities are evaluated at $\langle\phi\rangle=v_{X}$. The mass of $\phi$ is given by

$$
\begin{aligned}
m_{\phi}^{2} & =\left.\frac{d^{2} V}{d \phi^{2}}\right|_{\phi=v_{X}}=\overline{\beta_{\Phi}} v_{X}^{2} \\
& =\frac{6}{\pi} \overline{\alpha_{X}} m_{Z^{\prime}}^{2}\left(1-2\left(\frac{m_{N}}{m_{Z^{\prime}}}\right)^{4}\right),
\end{aligned}
$$

where $\alpha_{X}=g_{X}^{2} /(4 \pi)$. The condition for the stability of the $U(1)_{X}$ vacuum, $m_{\phi}^{2}>0$, requires $m_{Z^{\prime}}>2^{1 / 4} m_{N}$.

The $\mathrm{U}(1)_{X}$ gauge symmetry breaking by $\langle\Phi\rangle=v_{X} / \sqrt{2}$ induces a negative mass squared for the SM Higgs doublet $\left(-\lambda_{\text {mix }}|\langle\Phi\rangle|^{2}\right)$ in Eq. (2) and triggers the electroweak symmetry breaking [11]. The SM(-like) Higgs boson mass $\left(m_{h}=125 \mathrm{GeV}\right)$ is described as

\footnotetext{
${ }^{2}$ In our analysis, only a nonzero $\lambda_{\text {mix }} \ll 1$ at $v_{X}$ is important. Its contribution to the inflaton potential at high energies through the $\mathrm{RG}$ evolution is negligibly small [9].
} 


$$
m_{h}^{2}=\lambda_{\text {mix }} v_{X}^{2}=2 \lambda_{H} v_{h}^{2},
$$

where $v_{h}=246 \mathrm{GeV}$ is the Higgs doublet VEV. From this formula, we can justify our assumption of $\lambda_{\text {mix }} \ll 1$ by considering the LEP constraint on $v_{X} \gtrsim 10 \mathrm{TeV}$ [16-19].

The mass matrix for the Higgs bosons, $\phi$ and $h$, is given by

$$
\mathcal{L} \supset-\frac{1}{2}\left[\begin{array}{ll}
h & \phi
\end{array}\right]\left[\begin{array}{cc}
m_{h}^{2} & \lambda_{\text {mix }} v_{X} v_{h} \\
\lambda_{\text {mix }} v_{X} v_{h} & m_{\phi}^{2}
\end{array}\right]\left[\begin{array}{l}
h \\
\phi
\end{array}\right] .
$$

We diagonalize the mass matrix by

$$
\left[\begin{array}{l}
h \\
\phi
\end{array}\right]=\left[\begin{array}{cc}
\cos \theta & \sin \theta \\
-\sin \theta & \cos \theta
\end{array}\right]\left[\begin{array}{l}
\tilde{h} \\
\tilde{\phi}
\end{array}\right]
$$

where $\tilde{h}$ and $\tilde{\phi}$ are the mass eigenstates, and the mixing angle $\theta$ is determined by

$$
2 v_{X} v_{h} \lambda_{\text {mix }}=\left(m_{h}^{2}-m_{\phi}^{2}\right) \tan 2 \theta .
$$

Since we are interested in the case with $m_{\phi}^{2} \ll m_{h}^{2}$ and $\lambda_{\text {mix }} \ll 1$, we find

$$
\theta \simeq \frac{v_{h}}{v_{X}}=\frac{\sqrt{16 \pi \overline{\alpha_{X}}} v_{h}}{m_{Z^{\prime}}} \ll 1 .
$$

The mass eigenvalues are given by

$$
\begin{aligned}
& m_{\tilde{\phi}}^{2}=m_{\phi}^{2}+\left(m_{\phi}^{2}-m_{h}^{2}\right) \frac{\sin ^{2} \theta}{1-2 \sin ^{2} \theta} \simeq m_{\phi}^{2}-m_{h}^{2} \theta^{2}, \\
& m_{\tilde{h}}^{2}=m_{h}^{2}-\left(m_{\phi}^{2}-m_{h}^{2}\right) \frac{\sin ^{2} \theta}{1-2 \sin ^{2} \theta} \simeq m_{h}^{2} .
\end{aligned}
$$

For the parameter region that will be searched by FASER, we find $m_{\tilde{\phi}, \tilde{h}} \simeq m_{\phi, h}$ and $\tilde{\phi}, \tilde{h} \simeq \phi, h$. For notational simplicity, we will refer to the mass eigenstates without using tildes in the rest of this paper. Note that for a fixed value of $m_{N} / m_{Z^{\prime}}$, the inflaton mass $m_{\phi}$ and its mixing angle with the Higgs field $\theta$ are uniquely determined by $\overline{\alpha_{X}}$ and $m_{Z^{\prime}}$ with Eqs. (6) and (11).

\section{NONMINIMAL QUARTIC INFLATION}

Here we give a brief review on nonminimal quartic inflation with the action in the Jordan frame:

$\mathcal{S}_{J}=\int d^{4} x \sqrt{-g}\left[-\frac{1}{2} f(\phi) \mathcal{R}+\frac{1}{2} g^{\mu \nu}\left(\partial_{\mu} \phi\right)\left(\partial_{\nu} \phi\right)-V_{J}(\phi)\right]$,

where $\phi$ is a real scalar field (inflaton), $f(\phi)=\left(1+\xi \phi^{2}\right)$ with a real parameter $\xi>0, V_{J}(\phi)=\lambda \phi^{4} / 4$ is the inflaton quartic potential, and the reduced Planck mass of $M_{P}=2.44 \times 10^{18} \mathrm{GeV}$ is set to be 1 (Planck unit). Using the transformation of $f(\phi) g_{\mu \nu}=g_{E \mu \nu}$, the action in the Einstein frame is described as

$S_{E}=\int d^{4} x \sqrt{-g_{E}}\left[-\frac{1}{2} \mathcal{R}_{E}+\frac{1}{2} g_{E}^{\mu \nu}\left(\partial_{\mu} \sigma\right)\left(\partial_{\nu} \sigma\right)-V_{E}(\phi(\sigma))\right]$,

where $V_{E}(\phi(\sigma))=V_{J}(\phi) / f(\phi)^{2}$, and $\sigma$ is a canonically normalized scalar field (inflaton in the Einstein frame) which is related to the original field $\phi$ by

$$
\left(\frac{d \sigma}{d \phi}\right)^{2}=\frac{1+\xi(6 \xi+1) \phi^{2}}{\left(1+\xi \phi^{2}\right)^{2}}
$$

Using Eq. (15), we can express the slow-roll inflation parameters in the Einstein frame as

$$
\begin{aligned}
\epsilon(\phi)= & \frac{1}{2}\left(\frac{V_{E}^{\prime}}{V_{E} \sigma^{\prime}}\right)^{2}, \\
\eta(\phi)= & \frac{V_{E}^{\prime \prime}}{V_{E}\left(\sigma^{\prime}\right)^{2}}-\frac{V_{E}^{\prime} \sigma^{\prime \prime}}{V_{E}\left(\sigma^{\prime}\right)^{3}}, \\
\zeta(\phi)= & \left(\frac{V_{E}^{\prime}}{V_{E} \sigma^{\prime}}\right)\left(\frac{V_{E}^{\prime \prime \prime}}{V_{E}\left(\sigma^{\prime}\right)^{3}}-3 \frac{V_{E}^{\prime \prime} \sigma^{\prime \prime}}{V_{E}\left(\sigma^{\prime}\right)^{4}}\right. \\
& \left.+3 \frac{V_{E}^{\prime}\left(\sigma^{\prime \prime}\right)^{2}}{V_{E}\left(\sigma^{\prime}\right)^{5}}-\frac{V_{E}^{\prime} \sigma^{\prime \prime \prime}}{V_{E}\left(\sigma^{\prime}\right)^{4}}\right),
\end{aligned}
$$

where a prime denotes a derivative with respect to $\phi$. The slow-roll inflation takes place when $\epsilon,|\eta|, \zeta \ll 1$. The amplitude of the curvature perturbation,

$$
\Delta_{\mathcal{R}}^{2}=\left.\frac{V_{E}(\phi)}{24 \pi^{2} \epsilon(\phi)}\right|_{k_{0}},
$$

should satisfy $\Delta_{\mathcal{R}}^{2}=2.099 \times 10^{-9}$ from the Planck 2018 result [5] for the pivot scale $k_{0}=0.05 \mathrm{Mpc}^{-1}$. The number of $e$-folds is evaluated as

$$
N_{0}=\frac{1}{\sqrt{2}} \int_{\phi_{\mathrm{e}}}^{\phi_{0}} d \phi \frac{\sigma^{\prime}}{\sqrt{\epsilon(\phi)}},
$$

where $\phi_{0}$ is the inflaton value at the horizon exit of the scale corresponding to $k_{0}$, and $\phi_{e}$ is the inflaton value at the end of inflation, which is defined by $\epsilon\left(\phi_{e}\right)=1$. In our analysis, we fix $N_{0}=50$ to solve the horizon and flatness problems.

The inflationary predictions for the scalar spectral index $n_{s}$, the tensor-to-scalar ratio $r$, and the running of the spectral index $\alpha=\frac{d n_{s}}{d \ln k}$ are given by

$n_{s}=1-6 \epsilon+2 \eta, \quad r=16 \epsilon, \quad \alpha=16 \epsilon \eta-24 \epsilon^{2}-2 \zeta$, 
TABLE II. Inflationary predictions for various $\xi$ values and $N_{0}=50$. The region $\xi<0.00642(r>0.064)$ is excluded by the Planck 2018 result.

\begin{tabular}{lcccccc}
\hline \hline$\xi$ & $\phi_{0} / M_{p}$ & $\phi_{e} / M_{p}$ & $n_{s}$ & \multicolumn{1}{c}{$r$} & $\alpha\left(10^{-4}\right)$ & $\lambda$ \\
\hline 0 & 20.2 & 2.83 & 0.941 & 0.314 & -11.5 & $2.45 \times 10^{-13}$ \\
0.00527 & 20.05 & 2.77 & 0.954 & 0.1 & -9.74 & $7.83 \times 10^{-13}$ \\
0.00978 & 19.84 & 2.73 & 0.957 & 0.064 & -9.06 & $1.26 \times 10^{-13}$ \\
0.119 & 15.75 & 2.06 & 0.961 & 0.010 & -7.70 & $1.97 \times 10^{-12}$ \\
1 & 7.82 & 1.00 & 0.962 & 0.0049 & -7.51 & $6.56 \times 10^{-10}$ \\
10 & 2.65 & 0.337 & 0.962 & 0.0043 & -7.49 & $5.70 \times 10^{-8}$ \\
\hline \hline
\end{tabular}

which are evaluated at $\phi=\phi_{0}$. Using $\Delta_{\mathcal{R}}^{2}=2.099 \times 10^{-9}$ and $N_{0}=50$, the inflationary predictions for $\lambda, \phi_{0}$, and $\phi_{e}$ are determined as functions of the nonminimal gravitational coupling $\xi$. Based on unitarity arguments [20], we only consider $\xi<10$. Our results are summarized in Table II.

\section{NONMINIMAL U $(1)_{X}$ HIGGS INFLATON}

By introducing the nonminimal gravitational coupling of $-\xi\left(\Phi^{\dagger} \Phi\right) \mathcal{R}$, we identify the $\mathrm{U}(1)_{X}$ Higgs field with the inflaton field in Eq. (13). Since $\phi \gg v_{X}$ during inflation, we approximate the Higgs potential by its quartic potential in the following inflationary analysis.

For the inflation analysis, we employ the renormalization group (RG) improved effective potential of the form [21]

$$
V(\phi)=\frac{1}{4} \lambda_{\Phi}(\phi) \phi^{4},
$$

where $\lambda_{\Phi}(\phi)$ is the solution to the following RG equations at the one-loop level:

$$
\begin{aligned}
\frac{d \lambda_{\Phi}}{d \ln \phi}=\beta_{\lambda} \simeq 96 \alpha_{X}^{2}-3 \alpha_{Y}^{2}, \\
\frac{d \alpha_{X}}{d \ln \phi}=\beta_{g}=\frac{72+64 x_{H}+41 x_{H}^{2}}{12 \pi} \alpha_{X}^{2}, \\
\frac{d \alpha_{Y}}{d \ln \phi}=\beta_{Y}=\frac{1}{2 \pi} \alpha_{Y}\left(\frac{5}{2} \alpha_{Y}-6 \alpha_{X}\right) .
\end{aligned}
$$

Here, $\alpha_{Y}=Y_{M}^{2} /(4 \pi)$ and we have identified $\phi$ with the renormalization scale along the inflation trajectory.

Since $\lambda_{\Phi} \ll 1$, the stationary condition in Eq. (5) implies that $g_{X}, Y_{M} \ll 1$. Hence, the RG evolutions of $\alpha_{X}$ and $\alpha_{Y}$ can be approximated as

$$
\alpha_{X, Y}(\phi) \simeq \overline{\alpha_{X, Y}}+\overline{\beta_{g, Y}} \ln \left[\frac{\phi}{v_{X}}\right],
$$

and, accordingly,

$$
\beta_{\lambda}(\phi) \simeq \overline{\beta_{\lambda}}+2\left(96 \overline{\alpha_{X}} \overline{\beta_{g}}-3 \overline{\alpha_{Y}} \overline{\beta_{Y}}\right) \ln \left[\frac{\phi}{v_{X}}\right] .
$$

We now approximate the evolution of the quartic coupling as

$$
\begin{aligned}
& \lambda_{\Phi}(\phi) \simeq\left(\frac{11}{6}+\ln \left[\frac{\phi}{v_{X}}\right]\right) \bar{\beta}_{\lambda} \\
& \quad+\left(96 \overline{\alpha_{X}} \overline{\beta_{g}}-3 \overline{\alpha_{Y}} \overline{\beta_{Y}}\right)\left(\ln \left[\frac{\phi}{v_{X}}\right]\right)^{2} .
\end{aligned}
$$

In the following analysis, we fix $m_{N}=m_{Z^{\prime}} / 3$ (or, equivalently, $\overline{\alpha_{Y}}=8 \overline{\alpha_{X}} / 9$ ) to satisfy the vacuum stability condition. ${ }^{3}$ Using Eq. (24), the quartic coupling is determined as a function of $\phi, \overline{\alpha_{X}}, m_{Z^{\prime}}$, and $x_{H}$. On the other hand, in the inflation analysis, the inflationary predictions are controlled by only one parameter, $\xi$. Once we fix a $\xi$ value, $\phi_{0}$ and $\lambda_{\Phi}\left(\phi_{0}\right)$ are completely fixed, as listed in Table II. Hence, by using Eq. (24) we can express $\overline{\alpha_{X}}$ as a function of $m_{Z^{\prime}}$ and $x_{H}$ for a fixed value of $\xi$. In fact, for $\xi \lesssim 10$, we find that $\overline{\alpha_{X}}$ is almost independent of $x_{H}$, and the $x_{H}$ dependence for inflationary predictions effectively drops off. Therefore, the inflationary predictions for $\overline{\alpha_{X}}$, $m_{Z^{\prime}}, m_{\phi}$, and $\theta$ are directly related with each other through Eqs. (6), (11), and (24).

The ATLAS and CMS collaborations have been searching for a narrow resonance at the LHC, and the most severe constraint on the $Z^{\prime}$ boson of our model was obtained by the search with dilepton final states. The ATLAS Collaboration recently reported their final result of the LHC Run-2 with $139 \mathrm{fb}^{-1}$ integrated luminosity [22]. Following the analysis in Ref. [23], we interpret the ATLAS result to obtain an upper bound on $\overline{g_{X}}$ as a function of $m_{Z^{\prime}}$ for a fixed $x_{H}$ value. In Fig. 1, we show our results for $x_{H}=-0.8,0$, and 10 (the diagonal black lines from top to bottom). The upper bounds depend on $x_{H}$ values and roughly scale as $\overline{g_{X}} /\left|x_{H}\right|$ for $\left|x_{H}\right| \gtrsim 3$, while we find that the LHC bound becomes weak for $x_{H} \sim-1$ [24]. In the figure, we also plot the contours for fixed $\xi$ values. For $x_{H}=0$, the horizontal blue lines from top to bottom correspond to $\xi=10,1.0,0.12$, and $9.8 \times$ $10^{-3}$ or, equivalently, $r=4.3 \times 10^{-3}, 4.9 \times 10^{-3}, 0.01$, and 0.064 , respectively. The red shaded region is excluded by the Planck 2018 measurement, $r>0.064$. As discussed above, the inflationary predictions are almost independent of $x_{H}$ for $\left|x_{H}\right|<10$ and the horizontal lines represent the results for any values of $x_{H}$ for $\left|x_{H}\right|<10$. Figure 1 indicates a complementarity between the LHC search for the $Z^{\prime}$ boson resonance and the inflationary predictions. Since the $Z^{\prime}$ boson production cross section is dominated by the narrow resonance point, the cross section is proportional to $g_{X}^{2}$ in the narrow decay width approximation. Hence, we naively expect the upper bound on $g_{X}^{2}$ to scale with luminosity, so that the future bound on $g_{X} \simeq g_{X}($ current $) \times \sqrt{\frac{139 / \mathrm{fb}}{\mathcal{L} / \mathrm{fb}}}$.

\footnotetext{
${ }^{3}$ As long as $m_{N}^{4} \ll m_{Z^{\prime}}^{4} / 2$, our results remain unchanged. See Eq. (6).
} 


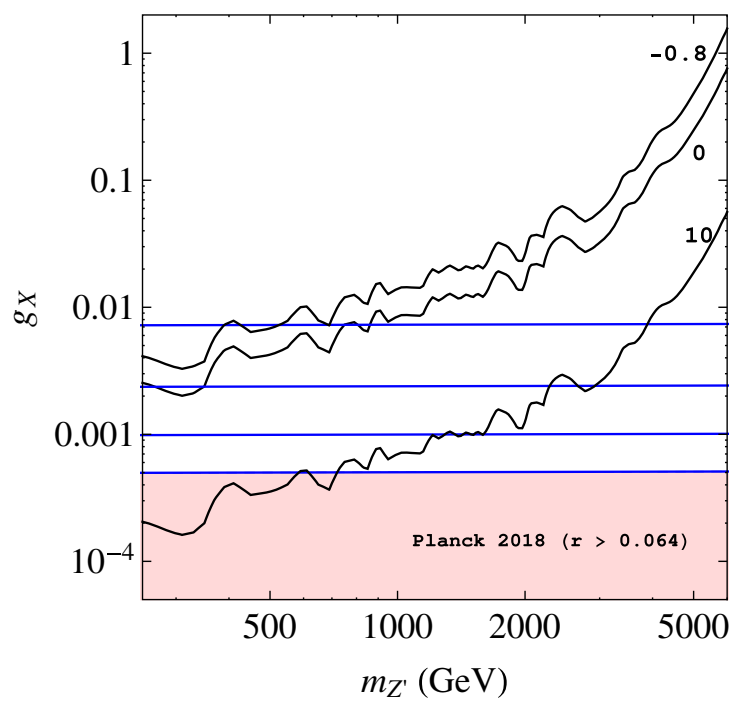

FIG. 1. Upper bounds on $\overline{g_{X}}$ from the ATLAS result for $x_{H}=-0.8,0$, and 10 (the diagonal black lines from top to bottom), respectively. For $x_{H}=0$, the horizontal blue lines from top to bottom correspond to $\xi=10,1.0,0.12$, and $9.8 \times 10^{-3}$ or, equivalently, $r=4.3 \times 10^{-3}, 4.9 \times 10^{-3}, 0.01$, and 0.064 , respectively. The red shaded region is excluded by the Planck 2018 measurements.

\section{SEARCHING FOR THE INFLATON AT FASER}

We are now ready to discuss the inflaton search at FASER and its complementarity to the cosmological constraints on the inflationary predictions. For a fixed $\xi$ value, the inflationary predictions are fixed and $\overline{\alpha_{X}}$ is determined as a function of $m_{Z^{\prime}}$, independently of $x_{H}$ for $\left|x_{H}\right|<10$. As a result, both the mass of the inflaton $m_{\phi}$ and its mixing angle with the SM Higgs field $\theta$ are uniquely determined by the CW relations in Eqs. (6) and (11), respectively.

In Fig. 2, we show our results ${ }^{4}$ in the $\left(m_{\phi}, \theta\right)$ plane, together with the FASER search reach, the search reach of other planned/proposed experiments (contours with the names of experiments indicated), and the current excluded region (gray shaded) from the CHARM [25], ${ }^{5}$ Belle [28], and $\mathrm{LHCb}$ [29] experiments, as shown in Ref. [3]. Here, to ensure the readability of Fig. 2, we have not shown the search reach of other experiments, such as SHiP [30], MATHUSLA [31], and CODEX-b [32] presented in Ref. [3]. After our analysis, each point in the FASER parameter space has a one-to-one correspondence with inflationary predictions and $Z^{\prime}$ boson search parameters. The diagonal dashed lines correspond to $\xi=9.8 \times 10^{-3}$

\footnotetext{
${ }^{4}$ The search reach and current experimental bound presented in Fig. 2 apply to any SM singlet scalar field.

${ }^{5}$ The updated analysis in Ref. [26] showed that the bound on $\theta$ for $m_{\phi} \simeq 0.3 \mathrm{GeV}$ could be significantly relaxed. Also, see Ref. [27] for recently updated bounds for $m_{\phi}=\mathcal{O}(0.1) \mathrm{GeV}$.
}

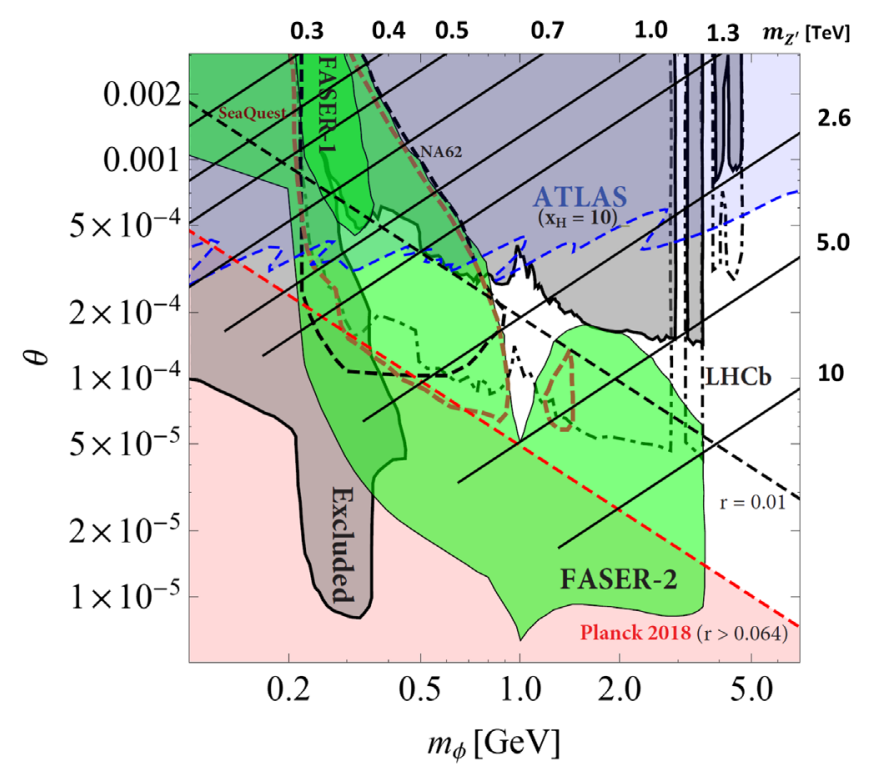

FIG. 2. Inflaton search reach at FASER (green shaded region) and the relation with other observables. The diagonal dashed lines correspond to $\xi=9.8 \times 10^{-3}(r=0.064)$ and $\xi=0.12$ $(r=0.01)$, respectively, from left to right. The diagonal solid lines correspond to $m_{Z^{\prime}}[\mathrm{TeV}]=0.3,0.4,0.5,0.7,1.0,1.3,2.6$, 5.0 , and 10, from top to bottom. The blue shaded region (labeled ATLAS) is excluded by the ATLAS result of the $Z^{\prime}$ boson search for $x_{H}=10$, corresponding to the bottom solid line in Fig. 1. The red shaded region is excluded by the Planck 2018 measurements.

( $r=0.064)$ and $\xi=0.12(r=0.01)$, respectively, from left to right. The light red shaded region $(r>0.064)$ is excluded by the Planck 2018 results. We find that the parameter region corresponding to the inflationary prediction $r \gtrsim 0.01$ can be searched by FASER 2 in the future, a part of which is already excluded the Planck 2018 result. For a fixed $m_{Z^{\prime}}$, we can obtain a relation between $m_{\phi}$ and $\theta$ through $\overline{\alpha_{X}}$ (recall, again, that this relation is almost independent of $x_{H}$ values for $\left|x_{H}\right|<10$ ). In Fig. 2, the diagonal solid lines correspond to $m_{Z^{\prime}}[\mathrm{TeV}]=0.3,0.4$, $0.5,0.7,1.0,1.3,2.6,5.0$, and 10 , from top to bottom. A point on a solid line corresponds to a fixed value of $\xi$ or, equivalently, $r$. Along each line, the $\xi(r)$ value increases (decreases) from left to right. For example, the left (right) diagonal dashed lines denote $r=0.0064$ and $r=0.01$. In Table III, for various $m_{Z^{\prime}}$ values, we list the range of the predicted tensor-to-scalar ratio $r$ that will be covered by

TABLE III. The ranges of $r$ that will be covered by FASER.

\begin{tabular}{lc}
\hline \hline$m_{Z^{\prime}}[\mathrm{TeV}]$ & The range covered by FASER \\
\hline 0.7 & $4.2 \times 10^{-3} \leq r \leq 9.0 \times 10^{-3}$ \\
1.0 & $8.4 \times 10^{-3} \leq r \leq 1.7 \times 10^{-2}$ \\
1.3 & $2.6 \times 10^{-3} \leq r \leq 9.9 \times 10^{-3}$ \\
2.6 & $1.3 \times 10^{-2} \leq r \leq 6.4 \times 10^{-2}$ \\
5.0 & $7.3 \times 10^{-3} \leq r \leq 6.4 \times 10^{-2}$ \\
10 & $1.1 \times 10^{-2} \leq r \leq 6.4 \times 10^{-2}$ \\
\hline \hline
\end{tabular}


FASER. The blue shaded region (labeled ATLAS) is excluded by the ATLAS result of the $Z^{\prime}$ boson search for $x_{H}=10$, corresponding to the bottom solid line in Fig. 1. The excluded regions for $x_{H}=-0.8$ and $x_{H}=0$ (the $B-L$ model limit) correspond to $\theta>10^{-3}$, and thus they are covered by the gray shaded region.

\section{INFLATON DECAY AND REHEAT TEMPERATURE}

To complete our discussion of the inflation scenario, let us now discuss reheating after inflation which proceeds via the decay of the inflaton to SM particles. In our case, the inflaton decays into light SM fermions through the mixing with the SM Higgs boson. Using Eq. (8), the decay width of the inflaton into SM particles can be expressed as

$$
\Gamma(\phi \rightarrow \mathrm{SM}) \simeq \theta^{2} \times \Gamma_{\mathrm{SM}}\left(m_{\phi}\right),
$$

respectively, where $\Gamma_{\mathrm{SM}}\left(m_{\phi}\right)$ is the total decay width of the SM Higgs boson if its mass were $m_{\phi}$. Using the inflaton decay width, we estimate the reheat temperature as

$$
T_{\mathrm{R}}=\left(\frac{90}{\pi^{2} g_{*}}\right)^{1 / 4} \sqrt{\Gamma(\phi \rightarrow \mathrm{SM}) \times M_{P}},
$$

where $g_{*}$ is the total effective degrees of freedom of the thermal plasma. Therefore, the reheat temperature is also determined by the two parameters $m_{\phi}$ and $\theta$ used in the preceding analysis. Both $m_{\phi}$ and $\theta$ are uniquely determined by $\xi, x_{H}$, and $m_{Z^{\prime}}$ values. In Fig. 3 , we show $m_{\phi}$ as a function of $x_{H}$ for fixed $m_{Z^{\prime}}=2 \mathrm{TeV}$. The horizontal solid lines from top to bottom correspond to $\xi=10,1.0$, and $9.8 \times 10^{-3}$ or, equivalently, $r=4.3 \times 10^{-3}, 4.9 \times 10^{-3}$, and 0.064 , respectively. We see that $m_{\phi}$ is independent of $x_{H}$ for $x_{H} \leq 10$.

In Fig. 4 , we show $T_{R}$ as a function of $m_{\phi}$ for fixed $x_{H}=0$. The lines from left to right correspond to contours for fixed $m_{Z^{\prime}}[\mathrm{TeV}]=0.7,1.3,2.6,5.0$, and 10 , respectively. The red shaded region corresponds to

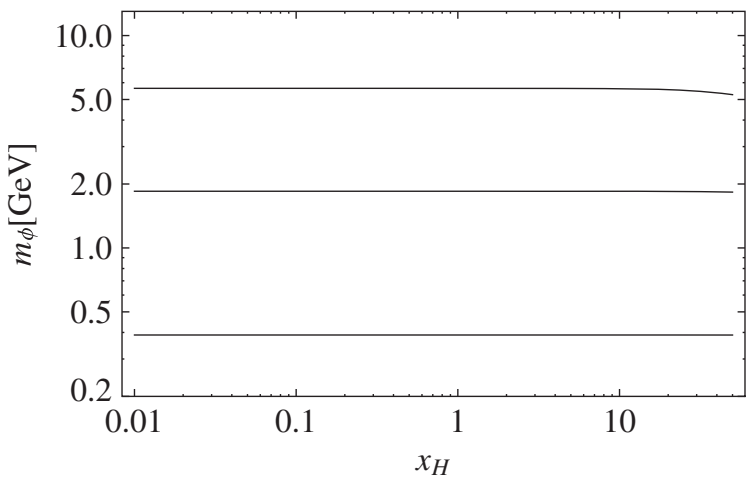

FIG. 3. For fixed $m_{Z^{\prime}}=2 \mathrm{TeV}$, the horizontal solid lines from top to bottom correspond to $\xi=10,1.0$, and $9.8 \times 10^{-3}$ or, equivalently, $r=4.3 \times 10^{-3}, 4.9 \times 10^{-3}$, and 0.064 , respectively. $\xi=9.8 \times 10^{-3} \quad(r>0.064)$ and is excluded by the Planck 2018 measurements. The dashed line corresponds to $\xi=0.12(r=0.01)$. For each contour in Fig. 4, we have constrained the inflaton mass to lie in the range $0.1<$ $m_{\phi}[\mathrm{GeV}] \leq 5$ and the mixing angle satisfies $\theta<10^{-3}$. For a fixed value of $m_{\phi}$, there is a one-to-one correspondence between the reheat temperature $T_{R}$ in Fig. 4 and the mixing angle $\theta$ in Fig. 2. Let us approximate the decay width as

$$
\Gamma_{\mathrm{SM}}\left(m_{\phi}\right) \simeq \frac{N_{c}}{8 \pi}\left(\frac{m_{f}}{v_{h}}\right)^{2} m_{\phi},
$$

where $N_{c}$ is the color factor for the fermion and antifermion in the final state with a mass $m_{f}<m_{\phi} / 2$. Together with Eqs. (25) and (26), we obtain

$$
\theta \simeq \frac{8.23 \times 10^{-5}}{\sqrt{N}_{c}}\left(\frac{0.1}{m_{f}}\right)\left(\frac{T_{R}}{5}\right)\left(\frac{1}{\sqrt{m_{\phi}}}\right),
$$

where $m_{f}, T_{R}$, and $m_{\phi}$ are in units of GeV.

In our analysis we have considered the number of $e$-folds $N_{0}$ as a free parameter and fixed $N_{0}=50$. However, $N_{0}$ is determined by $T_{R}$ and the tensor-to-scalar ratio $r$ (or, equivalently, the value of Hubble parameter during the inflation) as

$$
N_{0} \simeq 49.21+\frac{1}{6}\left(\frac{r}{0.01}\right)+\frac{1}{3}\left(\frac{T_{R}}{100 \mathrm{GeV}}\right) .
$$

This is consistent with our choice $N_{0}=50$. We have also checked that the inflationary prediction for a fixed $\xi$ value weakly depends on $N_{0}$ values.

In conclusion, we have considered the nonminimal quartic inflation scenario in the minimal $\mathrm{U}(1)_{X}$ model with classical conformal invariance, where the inflaton is identified with the $\mathrm{U}(1)_{X}$ Higgs field. FASER can search for the inflaton when its mass and mixing angle with the SM Higgs field are in the ranges $0.1 \lesssim m_{\phi}[\mathrm{GeV}] \lesssim 4$ and

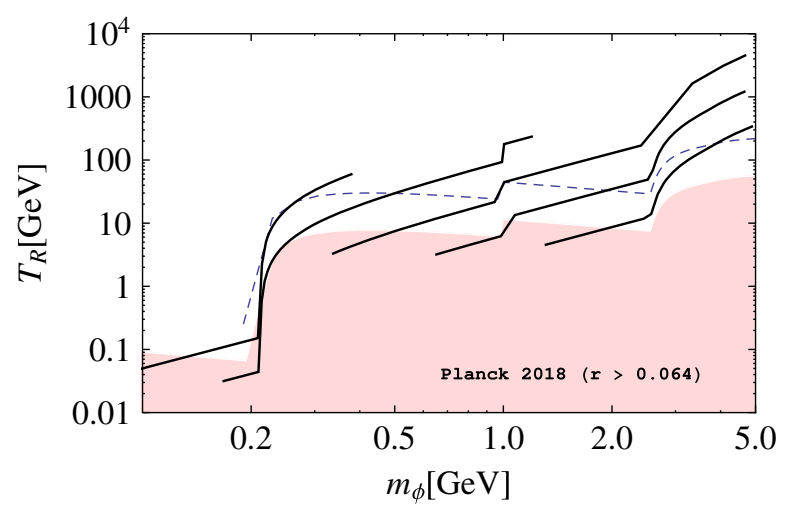

FIG. 4. For $x_{H}=0$, the solid lines from left to right correspond to $T_{R}$ contours for fixed $m_{Z^{\prime}}[\mathrm{TeV}]=0.7,1.3,2.6,5.0$, and 10 , respectively. The red shaded region is excluded by the Planck 2018 measurements corresponding to $r>0.064$ while the dashed lines correspond to $\xi=0.12(r=0.01)$. 
$10^{-5} \lesssim \theta \lesssim 10^{-3}$. By virtue of classical conformal invariance and the radiative $\mathrm{U}(1)_{X}$ symmetry breaking via the Coleman-Weinberg mechanism, the inflaton search by FASER, the $Z^{\prime}$ boson resonance search at the LHC, and the future measurement of $r$ are complementary tests of our inflationary scenario.

\section{ACKNOWLEDGMENTS}

This work is supported in part by the United States Department of Energy Grants No. DE-S0012447 (N. O.), No. DE-SC0013880 (D. R.) and Bartol Research Grant No. BART-462114 (D. R.).
[1] A. Ariga et al. (FASER Collaboration), FASER: ForwArd Search ExpeRiment at the LHC, arXiv:1901.04468; Technical Proposal for FASER: ForwArd Search ExpeRiment at the LHC, arXiv:1812.09139.

[2] J. L. Feng, I. Galon, F. Kling, and S. Trojanowski, Dark Higgs bosons at the ForwArd Search ExpeRiment, Phys. Rev. D 97, 055034 (2018).

[3] A. Ariga et al. (FASER Collaboration), FASERs physics reach for long-lived particles, Phys. Rev. D 99, 095011 (2019).

[4] F. Bezrukov and D. Gorbunov, Light inflaton Hunter's Guide, J. High Energy Phys. 05 (2010) 010.

[5] Y. Akrami et al. (Planck Collaboration), Planck 2018 results. X. Constraints on inflation, Astron. Astrophys. 641, A10 (2020).

[6] F. Bezrukov and D. Gorbunov, Light inflaton after LHC8 and WMAP9 results, J. High Energy Phys. 07 (2013) 140.

[7] S. Oda, N. Okada, D. Raut, and D. s. Takahashi, Nonminimal quartic inflation in classically conformal $\mathrm{U}(1)_{X}$ extended standard model, Phys. Rev. D 97, 055001 (2018).

[8] L. Marzola and A. Racioppi, Minimal but non-minimal inflation and electroweak symmetry breaking, J. Cosmol. Astropart. Phys. 10 (2016) 010.

[9] S. Oda, N. Okada, and D. s. Takahashi, Classically conformal U(1)' extended standard model and Higgs vacuum stability, Phys. Rev. D 92, 015026 (2015); A. Das, S. Oda, N. Okada, and D. s. Takahashi, Classically conformal U(1)' extended standard model, electroweak vacuum stability, and LHC Run-2 bounds, Phys. Rev. D 93, 115038 (2016).

[10] S. R. Coleman and E. J. Weinberg, Radiative corrections as the origin of spontaneous symmetry breaking, Phys. Rev. D 7, 1888 (1973).

[11] S. Iso, N. Okada, and Y. Orikasa, Classically conformal $B-L$ extended Standard Model, Phys. Lett. B 676, 81 (2009); The minimal B-L model naturally realized at TeV scale, Phys. Rev. D 80, 115007 (2009).

[12] W. A. Bardeen, On naturalness in the standard model, Report No. FERMILAB-CONF-95-391-T.

[13] R. Daido, F. Takahashi, and W. Yin, The ALP miracle: Unified inflaton and dark matter, J. Cosmol. Astropart. Phys. 05 (2017) 044; The ALP miracle revisited, J. High Energy Phys. 02 (2018) 104; F. Takahashi and W. Yin, ALP inflation and big bang on Earth, J. High Energy Phys. 07 (2019) 095.

[14] A. Davidson, $B^{-1}$ as the fourth color, quark-lepton correspondence, and natural masslessness of neutrinos within a generalized ws model, Phys. Rev. D 20, 776 (1979);
R. N. Mohapatra and R. E. Marshak, Local B-L Symmetry of Electroweak Interactions, Majorana Neutrinos and Neutron Oscillations, Phys. Rev. Lett. 44, 1316 (1980); Erratum, Phys. Rev. Lett. 44, 1643 (1980); Quark-lepton symmetry and B-L as the U(1) generator of the electroweak symmetry group, Phys. Lett. 91B, 222 (1980); C. Wetterich, Neutrino masses and the scale of B-L violation, Nucl. Phys. B187, 343 (1981); A. Masiero, J. F. Nieves, and T. Yanagida, $B^{-} 1$ violating proton decay and late cosmological baryon production, Phys. Lett. 116B, 11 (1982); R. N. Mohapatra and G. Senjanovic, Spontaneous breaking of global $B-L$ symmetry and matter-antimatter oscillations in grand unified theories, Phys. Rev. D 27, 254 (1983); W. Buchmuller, C. Greub, and P. Minkowski, Neutrino masses, neutral vector bosons and the scale of B-L breaking, Phys. Lett. B 267, 395 (1991).

[15] P. Minkowski, $\mu \rightarrow e \gamma$ at a rate of one out of $10^{9}$ muon decays?, Phys. Lett. 67B, 421 (1977); T. Yanagida, in Proceedings of the Workshop on the Unified Theory and the Baryon Number in the Universe, edited by O. Sawada and A. Sugamoto (KEK, Tsukuba, Japan, 1979), p. 95; M. Gell-Mann, P. Ramond, and R. Slansky, in Supergravity, edited by P. van Nieuwenhuizen et al. (North Holland, Amsterdam, 1979), p. 315; S. L. Glashow, The future of elementary particle physics, in Proceedings of the 1979 Cargèse Summer Institute on Quarks and Leptons, edited by M. Lévy et al. (Plenum Press, New York, 1980), p. 687; R. N. Mohapatra and G. Senjanović, Neutrino Mass and Spontaneous Parity Violation, Phys. Rev. Lett. 44, 912 (1980); J. Schechter and J. W. F. Valle, Neutrino masses in $\mathrm{SU}(2) \times \mathrm{U}(1)$ theories, Phys. Rev. D 22, 2227 (1980).

[16] LEP, ALEPH, DELPHI, L3, OPAL Collaborations, LEP Electroweak Working Group, SLD Electroweak Group, and SLD Heavy Flavor Group, A combination of preliminary electroweak measurements and constraints on the standard model, arXiv:hep-ex/0312023.

[17] M. Carena, A. Daleo, B. A. Dobrescu, and T. M. P. Tait, $Z^{\prime}$ gauge bosons at the tevatron, Phys. Rev. D 70, 093009 (2004).

[18] S. Schael et al. (ALEPH, DELPHI, L3, OPAL, and LEP Electroweak Collaborations), Electroweak measurements in electron-positron collisions at W-boson-pair energies at LEP, Phys. Rep. 532, 119 (2013).

[19] J. Heeck, Unbroken $B-L$ symmetry, Phys. Lett. B 739, 256 (2014).

[20] C. P. Burgess, H. M. Lee, and M. Trott, Power-counting and the validity of the classical approximation during inflation, 
J. High Energy Phys. 09 (2009) 103; C. P. Burgess, H. M. Lee, and M. Trott, Comment on Higgs inflation and naturalness, J. High Energy Phys. 07 (2010) 007; J. L. F. Barbon and J. R. Espinosa, On the naturalness of Higgs inflation, Phys. Rev. D 79, 081302 (2009); M. P. Hertzberg, On inflation with non-minimal coupling, J. High Energy Phys. 11 (2010) 023.

[21] For a review, see M. Sher, Electroweak Higgs potentials and vacuum stability, Phys. Rep. 179, 273 (1989).

[22] G. Aad et al. (ATLAS Collaboration), Search for high-mass dilepton resonances using $139 \mathrm{fb}^{-1}$ of $p p$ collision data collected at $\sqrt{s}=13 \mathrm{TeV}$ with the ATLAS detector, Phys. Lett. B 796, 68 (2019).

[23] A. Das, P. S. B. Dev, and N. Okada, Long-lived TeV-scale right-handed neutrino production at the LHC in gauged $U(1)_{X}$ model, Phys. Lett. B 799, 135052 (2019).

[24] N. Okada and S. Okada, $Z^{\prime}$-portal right-handed neutrino dark matter in the minimal $\mathrm{U}(1)_{X}$ extended Standard Model, Phys. Rev. D 95, 035025 (2017).

[25] F. Bergsma et al. (CHARM Collaboration), Search for axion like particle production in $400-\mathrm{GeV}$ proton-copper interactions, Phys. Lett. 157B, 458 (1985).

[26] M. W. Winkler, Decay and detection of a light scalar boson mixing with the Higgs boson, Phys. Rev. D 99, 015018 (2019).

[27] The MicroBooNE Collaboration, Search for a Higgs portal scalar decaying to electron-positron pairs in MicroBooNE, Report No. MICROBOONE-NOTE-1092-PUB; D. EganaUgrinovic, S. Homiller, and P. Meade, Light Scalars and the Koto Anomaly, Phys. Rev. Lett. 124, 191801 (2020);
S. Foroughi-Abari and A. Ritz, LSND constraints on the Higgs Portal, Phys. Rev. D 102, 035015 (2020); E. Cortina Gil et al. (NA62 Collaboration), Search for $\pi^{0}$ decays to invisible particles, G. Ruggiero, New result on $K^{+} \rightarrow \pi^{+} \nu \bar{\nu}$ from the NA62 experiment, J. Phys. Conf. Ser. 1526, 012003 (2020).

[28] J.-T. Wei et al. (Belle Collaboration), Measurement of the Differential Branching Fraction and Forward-Backword Asymmetry for $B \rightarrow K^{(*)} \ell^{+} \ell^{-}$, Phys. Rev. Lett. 103, 171801 (2009).

[29] R. Aaij et al. (LHCb Collaboration), Search for HiddenSector Bosons in $B^{0} \rightarrow K^{* 0} \mu^{+} \mu^{-}$Decays, Phys. Rev. Lett. 115, 161802 (2015); Search for long-lived scalar particles in $B^{+} \rightarrow K^{+} \chi\left(\mu^{+} \mu^{-}\right)$decays, Phys. Rev. D 95, 071101 (2017).

[30] S. Alekhin, W. Altmannshofer, T. Asaka, B. Batell, F. Bezrukov, K. Bondarenko, A. Boyarsky, K. Y. Choi, C. Corral, N. Craig et al., A facility to search for hidden particles at the CERN SPS: The SHiP physics case, Rep. Prog. Phys. 79, 124201 (2016).

[31] J. A. Evans, Detecting hidden particles with MATHUSLA, Phys. Rev. D 97, 055046 (2018); D. Curtin, M. Drewes, M. McCullough, P. Meade, R. N. Mohapatra, J. Shelton, B. Shuve, E. Accomando, C. Alpigiani, S. Antusch et al., Long-lived particles at the energy frontier: The MATHUSLA physics case, Rep. Prog. Phys. 82, 116201 (2019).

[32] V. V. Gligorov, S. Knapen, M. Papucci, and D. J. Robinson, Searching for long-lived particles: A compact detector for exotics at LHCb, Phys. Rev. D 97, 015023 (2018). 